\title{
Acceleration and Evolution of Cosmic Ray Electrons During Radio-Galaxy-Cluster-Shock Encounters
}

\author{
T. W. Jones* \\ University of Minnesota \\ E-mail: twj@umn.edu \\ B. J. O’Neill \\ University of Minnesota \\ E-mail: oneilleastro.umn.edu \\ C. Nolting \\ University of Minnesota \\ E-mail: nolt0040@umn.edu \\ P. J. Mendygral \\ Cray, Inc \\ E-mail: peter@mendygral.org
}

\begin{abstract}
Radio Galaxies (radio-loud AGNs, or RGs) are common in galaxy cluster media (ICMs). In addition to their potential thermodynamical roles, these RGs may also serve to illuminate important ICM dynamical features, especially ICM shocks. Those interactions are revealed most clearly by radio synchrotron emissions from associated RG Cosmic Ray Electrons (CRe). Here we report results from "MHD+CR" simulations of RG-shock encounters and specifically on resultant shock acceleration (DSA) of the $\mathbf{C R e}$ and observable consequences. We find, in particular, that even modest-strength ICM shocks $\left(M_{s} \sim 3-4\right)$ may lead to significant and observable DSA of the RG CRe. This work has impact on both the nature of large scale cluster radio emissions, including so-called radio relics and our understanding of RG-ICM-shock dynamics.
\end{abstract}

35th International Cosmic Ray Conference - ICRC2017

10-20 July, 2017

Bexco, Busan, Korea

\footnotetext{
* Speaker.
} 


\section{Introduction}

Radio Galaxies (RG) are common in clusters of galaxies. They represent the interaction between high velocity plasma jets formed by supermassive black holes in resident galaxies with the diffuse plasma filling the cluster, commonly known as the Intracluster Medium or ICM. If the ICM is quiescent and at rest with respect to the RG the jets excavate cavities in the ICM and fill them with RG plasma. The RG plasma includes relativistic, cosmic ray electrons, $\mathbf{C R e}$, as well as magnetic fields. Together they illuminate the cavities with radio synchrotron emission (see, for example, [1]). In reality, on the other hand, both individual galaxies and ICMs are generally in motion within a cluster. That can lead to substantial distortions in the RG cavities (see, for example [2]). In clusters that are highly disturbed, when, for instance, they are undergoing mergers, or when a RG falls into a cluster as part of accretion from a cosmic filament [3] the relative RG-ICM velocities can be significantly supersonic. During mergers RG-ICM interactions may then also commonly involve an ICM shock of moderate strength, since shocks with sonic Mach numbers $M \sim 2-4$ are expected well inside the cluster accretion radius. Such a shock encounter will substantially modify the pre-existing RG structure, and can even disrupt it. The encounters might also lead to enhanced synchrotron emissions due to compression and particle acceleration. In fact, such RG-shock encounters have been invoked to explain some of the giant, so-called "radio relics" seen in a number of merging clusters $[4,5,6,7]$. The basic physics of idealized shock- RG-cavity dynamical interactions has been treated before [4, 3]. However, as far as we are aware, there has not previously been a detailed, self-consistent examination of these encounters when the RG is a dynamically formed structure that contains both CRe and magnetic fields, and, in particular, an exploration of the consequent evolution of those $\mathbf{C R e}$ and magnetic fields and the resulting synchrotron emissions.

It turns out that there can be substantial differences in these behaviors in dynamically formed RG structures compared to static cavities. These differences center on the character of the external (ICM-based) shock as it penetrates through the RG plasma. To elucidate this point consider the ideal encounter between an external plane shock and a spherical cavity with an initially thin boundary. The two media are initially supposed to be in pressure balance. Since the density in the cavity is lower than in the ICM it must be hotter to produce an equal pressure. Thus, the sound speed in the cavity is generally higher than in the ICM. The shock, upon entering the cavity, will weaken because of the higher sound speed, but it will still propagate faster than in the external medium (see, for example, [3]). The faster post shock flow in the cavity interior, followed by post shock ICM, generates vorticity along the cavity perimeter. The original cavity is destroyed and replaced with a planar vortex ring with the ring axis aligned with the shock normal. Most important to our discussion here, the weakened interior shock is less effective at Diffusive Shock Acceleration (DSA) of the CRe. That is why, for instance, [4] assumed that CRe in the cavity would only experience adiabatic compression during shock passage. If, on the other hand the pre-shock state of the RG cavity has a complex boundary with, because of mixing, substantial regions of higher density than in the ideal cavity, then the propagation of the external shock through the RG structure can be more complex and can include regions where the shock is strong. Then, DSA can be more effective than in the ideal shock-cavity encounter.

Here we report some preliminary results from our efforts to conduct a study of these issues using high resolution 3D MHD simulations that include explicit CRe transport incorporating the 
physics of DSA. We have explored a variety of external, ICM shock strengths, pre-existing conditions for the RGs (for instance, RGs distorted into "Narrow Angle Tail" (NAT) formed in cross winds prior to their shock encounters), as well as a variety of orientations between the RG jets and the direction of shock propagation. Some general dynamical considerations were outlined previously [2]. Here we focus on issues associated with the evolution of CRe initially embedded in the RG jets during such encounters, along with their synchrotron signatures. Space here does not allow a full discussion, so we provide only some essential aspects of the simulations and preliminary findings on these issues.

\section{The Simulations}

Our simulations were carried out with the WOMBAT MHD code [8] on a uniform 3D Cartesian grid employing a $2^{\text {nd }}$ order TVD scheme with CT magnetic flux conservation. In the simulations discussed here the spatial resolution was $\Delta x=0.5 \mathrm{kpc}$. The RG jets were formed in a cylindrical volume within the simulation domain having a core radius $r_{j}=3 \mathrm{kpc}$, so six cells, surrounded by a thin transition layer to the ambient environment. The "jet-source" cylinder can be arbitrarily oriented on the grid. Here it was aligned with one of the principal grid axes, depending on the desired orientation with any imposed initial ambient ICM flow and with a planar ICM shocks of primary interest in our present discussion. The full grid was always made large enough and the jet source located in each case to contain all the dynamics associated with the RG flows and the responses within the ICM during the full simulation.

For the simulations outlined here domain dimensions ranged roughly $\sim 500 \mathrm{kpc}$ to $\sim 1 \mathrm{Mpc}$ in each direction. Bipolar, parallel jets were formed with uniform density and gas pressure in the jet source. In the cases discussed here the jet plasma density was $1 \%$ of the undisturbed ICM density, while the jet gas pressure was equal to the undisturbed ICM gas pressure. A toroidal magnetic field was formed in the jet source with a peak strength at $r_{j}$ corresponding to effective $\beta_{p}=P_{g} / P_{B} \sim$ $25-75$. The emergent jets had internal Mach numbers ${ }^{1}, M \sim 2.5-10$, and carried a passive $\mathbf{C R e}$ population with an initial power law spectrum $\left(f(p) \propto p^{-q_{0}}\right)$ with $q_{0}$ in the range 4.2 to 4.5. For reference below, the associated synchrotron spectral slope $\left(I_{v} \propto v^{-\alpha}\right)$ for a given $q$ is $\alpha=(q-$ 3) $/ 2$. The diffusion-convection equation for the CRe population was evolved over the full domain using our Eulerian, "Coarse-Grained Finite Momentum Volume scheme [9], including adiabatic and radiative energy gains and losses, as well as DSA in shocks. In a shock crossing the upstream CRe spectrum was evaluated and, if any momentum range, the upstream slope, $q>q_{M}$, with $q_{M}=4 M^{2} /\left(M^{2}-1\right), q$ was reset to $q_{M}$. We turned off any injection of fresh CRe at shocks, so that the only CRe involved were those that originated within the jet source. Further, in order to focus on the RG CRe and emissions, the ambient, homogeneous ICM was unmagnetized and devoid of CRe. Thus, synchrotron emissions could come only from regions that contain RG plasma. Dynamical mixing between the RG and ICM plasmas, in some situations, did cause significant initial ICM plasma to become magnetized and to include substantial CRe before the external shock encounter. Those regions then were possible sources of synchrotron emission. The properties of that emission were, of course, subsequently influenced by CRe energy gains and losses, including DSA.

\footnotetext{
${ }^{1}$ The jet velocities were in practice $\sim 0.1 \mathrm{c}$.
} 


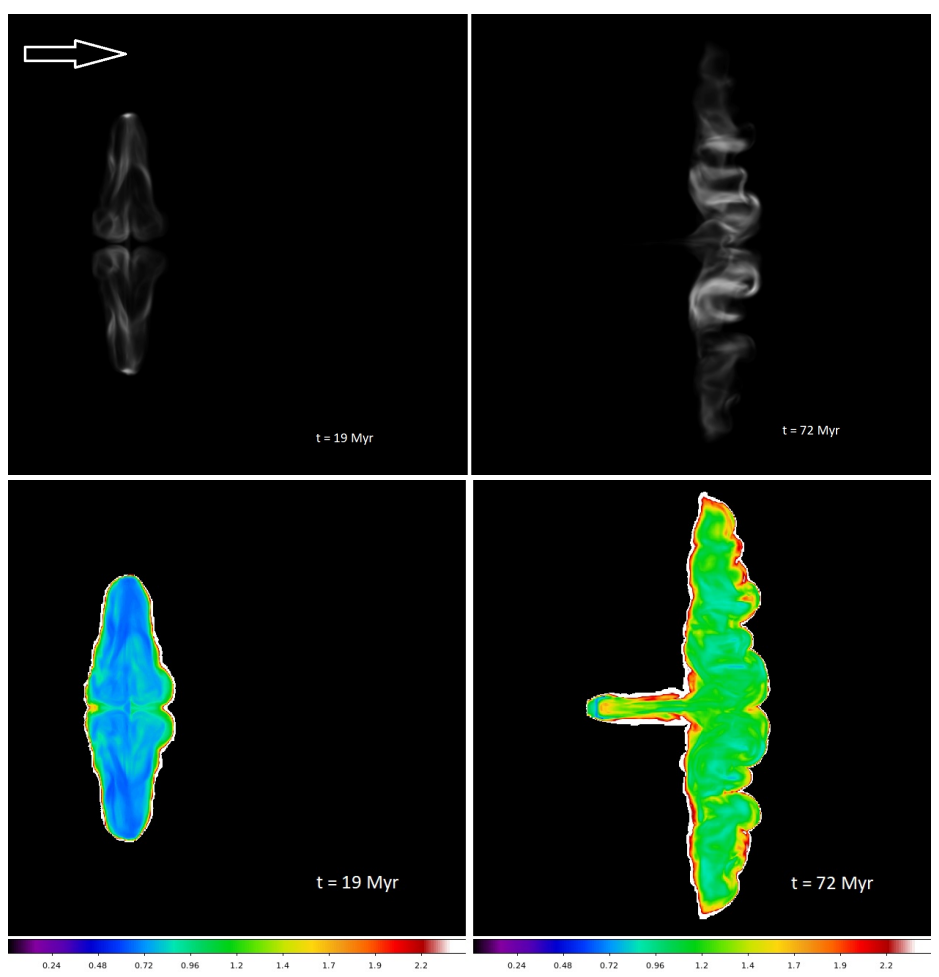

Figure 1: Top: Radio synchrotron brightness images ( $325 \mathrm{MHz}$ ) for Case 1 in which a pair of RG cavities are overrun by a Mach 4 ICM shock with normal orthogonal to the jet axis (Arrow shows shock motion). Line of sight is orthogonal to both the jets and the shock normal. Initial impact is at $t \approx 19 \mathrm{Myr}$ (left). The shocked RG cavities evolved into co-joined vortex rings, shown on the right approximately $50 \mathrm{Myr}$ after impact. Bottom: Radio synchrotron spectral index, $\alpha$, images $(600 \mathrm{MHz}-1.4 \mathrm{GHz})$ for the same two times. Spectral index keys are at the bottom of each image. The emergent jet $\alpha_{0}=0.6$ (blue), while the steepest spectra on the right have $\alpha \sim 1.5$ (yellow), and along some edges $\alpha \sim 2$ (red).

\section{Results}

Here we look briefly at two complementary situations we simulated. In Case 1, a Mach 10 jet pair with $\beta_{p}=75$ and $q_{0}=4.2$ (synchrotron index, $\alpha_{0}=0.6$ ), drove into a static ICM for approximately 19 Myr before being overrun by a Mach 4 shock propagating at right angles to the jet axis. Prior to the shock encounter the RG has the appearance of a classical, "FRII", double lobed radio source (see Fig. 1). As the shock penetrated the RG cavities, we turned off the jets in this case, leaving the two cavities isolated. The ICM shock very quickly passed through the low density RG. As in the ideal shock-cavity encounter, the shocked cavities each formed ring vortices, although the two vortices soon merged into one, elongated ring with major axis roughly coincident with the original jets. Here, as in the ideal case described above, the passage of the external, ICM shock did not result in detectable DSA re-acceleration of the RG CRe. Over time the CRe spectra progressively steepened due to radiative losses from inverse Compton scattering of the CMB and from synchrotron emission. This is evident in both the radio synchrotron spectral images shown in Fig. 1 and the example CRe distribution function within the RG at the same two times, as shown in Fig. 2. 

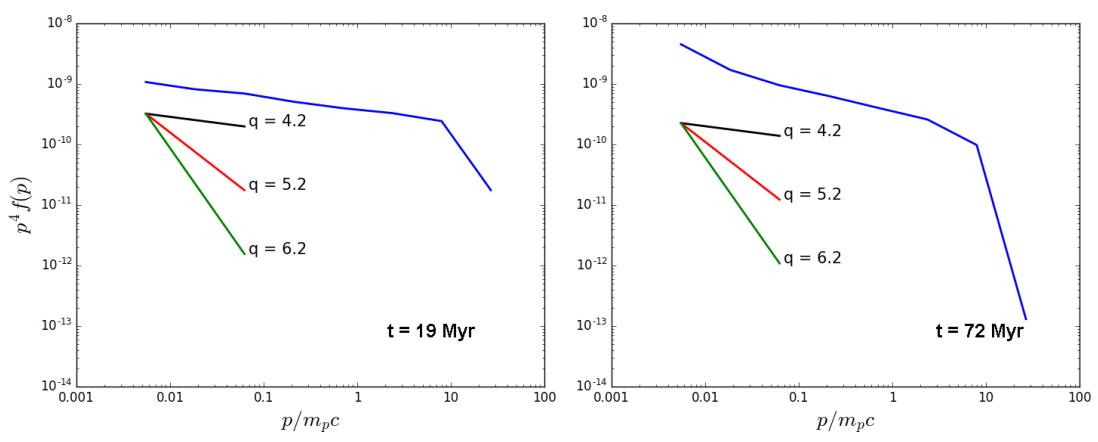

Figure 2: The CRe distribution function in Case $\mathbf{1}$ for a sample RG plasma element at the two times of Fig. 1. The emergent jet $q_{0}=4.2$. CRe momentum, $p$, has been normalized by proton rest mass, $m_{p} c$; $\gamma_{\mathrm{CRe}} \approx 1836 p / m_{p} c$.

In Case 2 a Mach 2.5 jet pair with $\beta_{p}=25$ and $q_{0}=4.5\left(\alpha_{0}=0.75\right)$ was placed in a steady Mach 0.75 cross wind in order to form a NAT radio structure prior to the ICM shock impact at right angles to the NAT axis. In the Fig. 3 radio image the cross wind comes from the left (purple arrow), while the jet axis (towards the left of the image) is vertical. Approximately $554 \mathrm{Myr}$ after the NAT began to form, with the tails by now $\sim 700 \mathrm{kpc}$ long, a planar Mach 3 ICM shock struck the lower tail from below (white arrow). At the time shown in Fig. 3 ( $t=574 \mathrm{Myr})$ the shock has passed through most of the lower tail, but has not reached the upper tail. Eventually the shock crosses both tails. Their relative separation is reduced and they would to most viewers appear almost as one structure.

Note in Fig. 3 that the synchrotron emission in the lower. shocked tail extends to much greater distances from the jet source than the upper, unshocked tail and also exhibits much flatter spectral slopes. CRe populations in the upper tail have "aged" so severely that their spectra are very steep; they have become "synchrotron dark" over most of the tail at radio frequencies. In the lower tail, on the other hand, shock compression has increased the CRe densities and the local magnetic field strengths, greatly enhancing the synchrotron brightness. The flatter spectra evident in the lower tail, contribute even more to the increased synchrotron brightness. The flattening results from significant DSA in some regions within the tail. That did not occur in the Case $\mathbf{1}$ simulation. What is the difference here?

Unlike the relatively "clean" RG lobes in Case 1 the NAT was highly turbulent before the shock encounter. This caused dynamical mixing between the RG and the ICM plasmas. Recall in these simulations that the ICM itself was made unmagnetized and absent any $\mathbf{C R e}$, so would not be a source of synchrotron emission. But, mixed regions could become radio bright. The turbulent tails also developed wide variations in total plasma density, so also in local sound speed. Thus, unlike the Case 1 scenario, the penetrating ICM shock developed a wide range of speeds and Mach numbers. That is quite evident in Fig, 4, which shows a volume rendering of the shock surface at the same time as the radio images in Fig. 3. Recall that the incident shock was Mach 3. In lower density portions of the tail the penetrating shock was weaker, as expected. But, in higher density regions it could be as strong or even stronger than the incident shock. The consequences to the CRe spectra are illustrated in Fig. 5, which shows the CRe momentum spectrum in a region roughly 


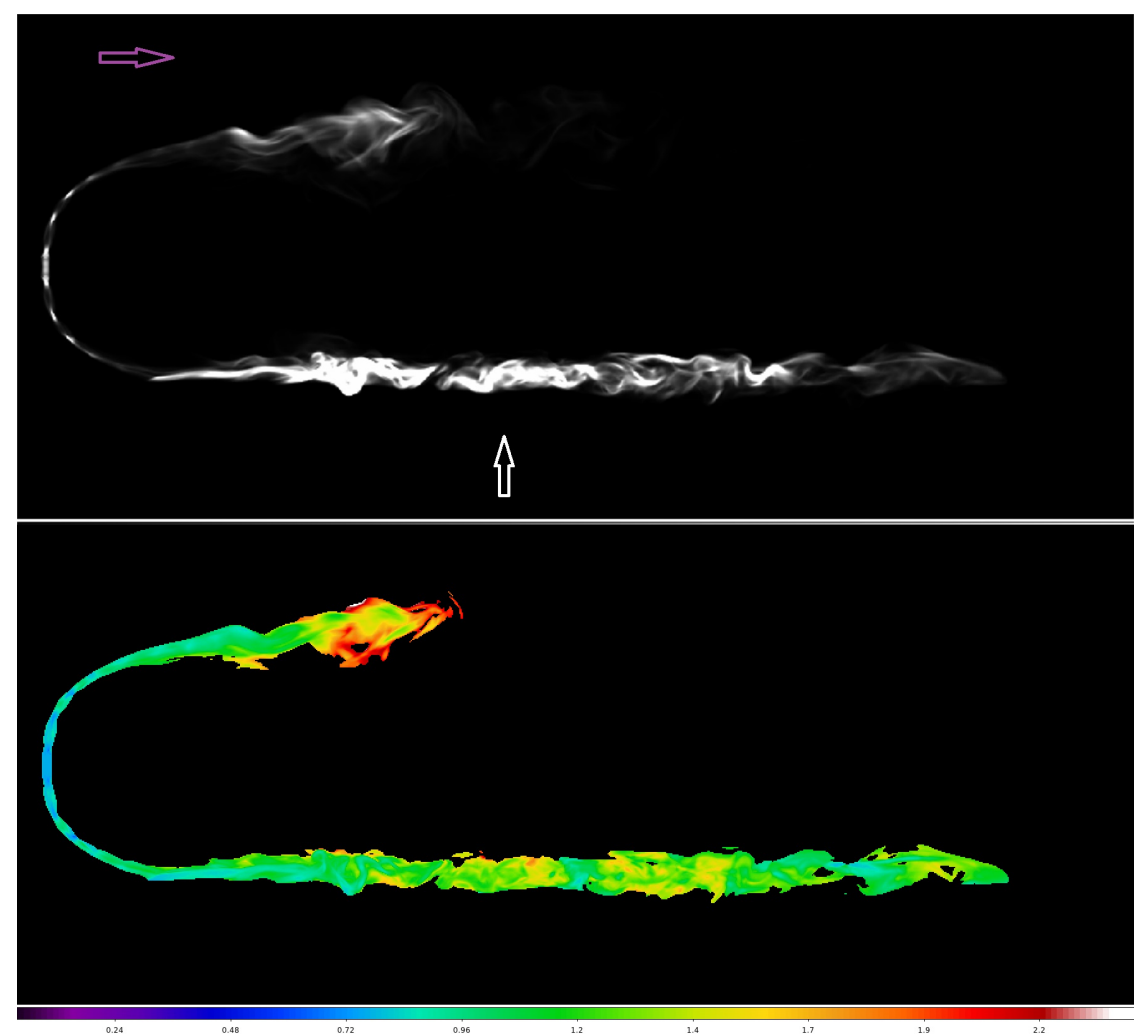

Figure 3: Upper: $175 \mathrm{MHz}$ radio image of the Case 2, NAT-shock encounter just as the $\mathrm{M}=3$ ICM shock crosses the lower of two NAT RG tails. Purple arrow indicates pre-shock cross wind direction; white arrow shows shock motion. Line of sight is orthogonal to the plane of the tails and to the shock normal. Lower: Radio spectral image of the same $(600 \mathrm{MHz}-1.4 \mathrm{GHz})$. Color code: cyan $(\alpha \sim 0.75)$. green $(\alpha \sim 1.2)$, yellow $(\alpha \sim 1.5)$, red $(\alpha \sim 2)$.

in the middle of the lower tail at two times. The purple line represents the spectrum just before shock passage. The green line shows the spectrum in the same volume just after shock passage. The spectrum for $\mathbf{C R e}$ with energies above about $1 \mathrm{GeV}$ has been flattened substantially by DSA in the shock. The dashed line illustrates the slope $q=5.24$ which corresponds to DSA in a shock with Mach number 4.25. The associated synchrotron spectral index is $\alpha \approx 1.1$, which actually corresponds roughly to the integrated synchrotron spectral index at this time of the tail below 1 GHz.

\section{Conclusions}

Encounters between RG plasmas and large scale ICM shocks should be relatively common occurrences in galaxy clusters. The encounters may strongly deform the distribution of RG plasma and greatly influence radio synchrotron emissions from embedded CRe. Here we have explored two complementary examples of such encounters, emphasizing important dependencies of the observable consequences on the history of the RG plasma prior to its encounter with the shock. Perhaps most importantly, the possibilities for significant $1^{\text {st }}$ order Fermi acceleration at the shock as it penetrates the RG plasma depend strongly on the density structure in RG plasma. That, in 


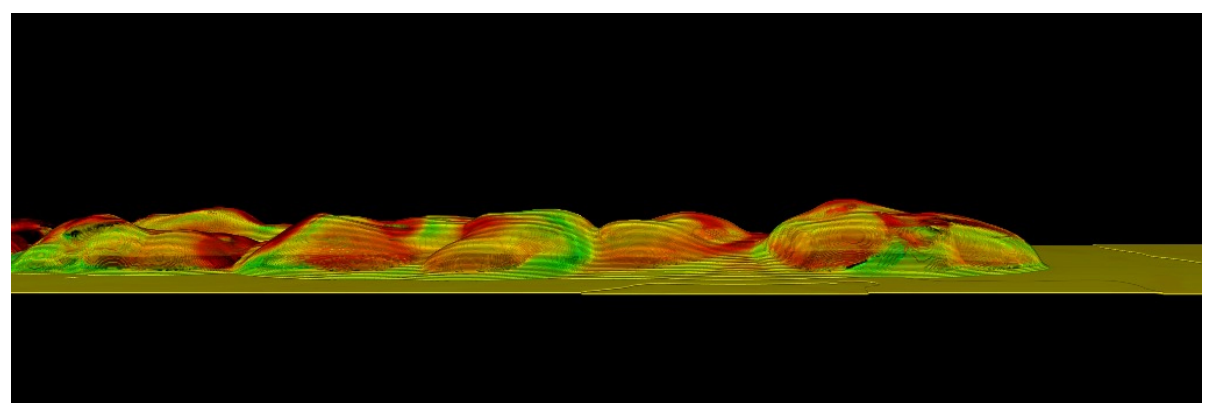

Figure 4: Volume rendering of the shock surface for Case 2 at the time shown in Fig. 3. Surface is color coded by Mach number: Mach $4=$ green, Mach $3=$ yellow (ICM value), Mach $2=$ red. The viewing direction is tilted about 20 degrees from the line of sight in Fig. 3 to make the geometry more apparent.

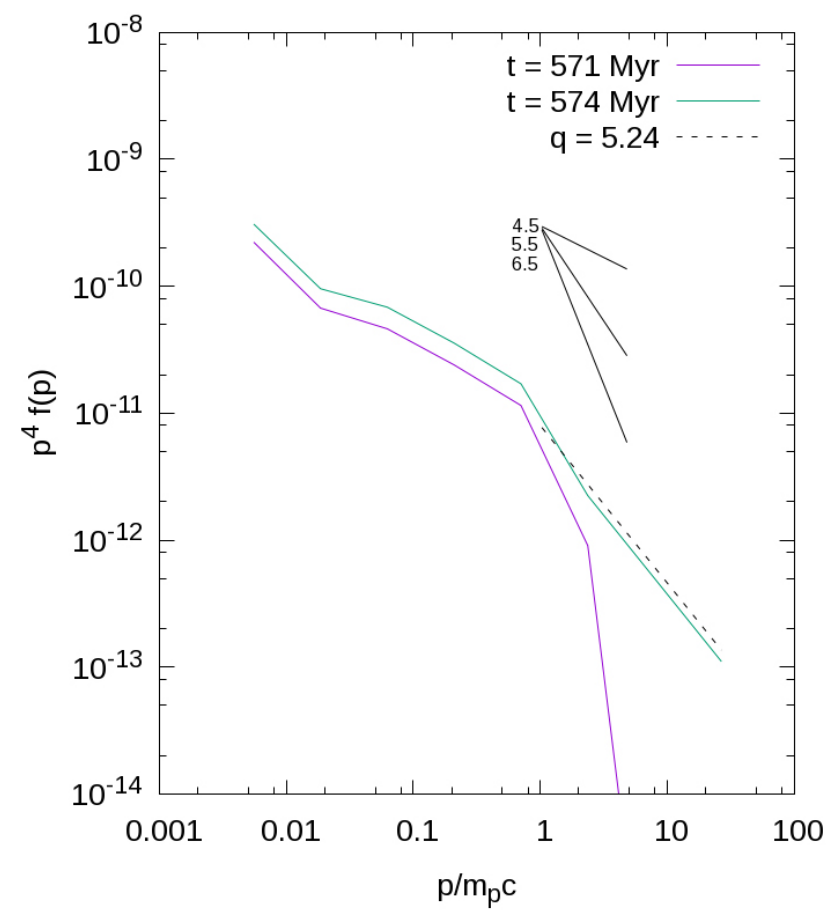

Figure 5: Fig 5 The CRe distribution function in Case 1 for a sample RG plasma element at the two times of Fig. 1. The emergent jet $q_{0}=4.2$.

turn may be strongly influenced by the degree of dynamical mixing between the two plasmas. In particular, if the RG can be simply represented as clean, low density cavities, then the shock will be weak inside the RG and unlikely to produce significant particle acceleration. On the other hand, if, through turbulent mixing, for instance, the RG contains significant regions of higher density, the shock can in those regions be strong enough to lead to particle acceleration that can significantly influence the appearance of the RG, as well as our interpretation of observations.

Acknowledgments This work at the University of Minnesota was supported in part by NSF grant AST1211595. The reported simulations utilized high performance resources made available by the University of Minnesota Supercomputing Institute. 


\section{References}

[1] M. S. Longair, High Energy Astrophysics, Cambridge University Press, Cambridge, 2011

[2] T. W. Jones, C. Nolting, B. J. O'Neill \& P, J. Mendygral, Using collisions of AGN outflows with ICM shocks as dynamical probes, $\mathrm{PhPl}, 24$ (2017) 1402

[3] C. Pfrommer \& T. W. Jones, Radio Galaxy NGC 1265 Unveils the Accretion Shock Onto the Perseus Galaxy Cluster, ApJ, 730 (2011) 22

[4] T. Ensslin \& M. Bruggen, On the formation of cluster radio relics, MNRAS, 331 (2002) 1011

[5] R. van Weeren, F. Andrade-Santos etal., The case for electron re-acceleration at galaxy cluster shocks, Nature Astronomy, 1, 5

[6] T. W. Shimwell, M. Markevitch, S. Brown, L. Feretti, B. M. Gaensler, M. Johnston-Hollitt, C. Lage \& R. Srinivasan, Another shock for the Bullet cluster, and the source of seed electrons for radio relics, MNRAS, 449 (2015) 1486

[7] H. Kang, D. Ryu \& T. W. Jones, Shock Acceleration Model for the Toothbrush Radio Relic, ApJ, 840 (2017) 42

[8] P. J. Mendygral, N. Radcliffe, K. Kandalla, D. Porter, N. J. O’Neill, C. Nolting, P. Edmon, J. M. Donnert \& T. W. Jones, WOMBAT: A Scalable and High-performance Astrophysical Magnetohydrodynamics Code, ApJS, 228 (2017) 23

[9] T. W. Jones \& H. Kang, An efficient numerical scheme for simulating particle acceleration in evolving cosmic-ray modified shocks, APh, 24 (2005) 75 